\title{
Post-procedural TIMI flow grade 2 is not associated with improved prognosis in patients with non-ST-segment elevation myocardial infarction undergoing percutaneous coronary revascularization (PL-ACS registry)
}

Jarosław Karwowski ${ }^{1}$, Lech Poloński ${ }^{2}$ Marek Gierlotka ${ }^{2}$, Mariusz Gąsior ${ }^{2}$, Maciej Bęćkowski ${ }^{1}$, Ilona Kowalik ${ }^{1}$, Hanna Szwed ${ }^{1}$

${ }^{1} 2^{\text {nd }}$ Department of Coronary Artery Disease, Institute of Cardiology, Warsaw, Poland

${ }^{2} 3^{\text {rd }}$ Department of Cardiology, Silesian Center for Heart Diseases, Zabrze, Poland

\section{Abstract}

Background: The impact of final Thrombolysis in Myocardial Infarction (TIMI) flow in the infarct-related artery (IRA) on outcomes in non-ST-segment elevation myocardial infarction (NSTEMI) patients treated with percutaneous coronary intervention (PCI) is unknown. This study aimed to evaluate the impact of post-procedural TIMI flow in IRA on outcomes in NSTEMI patients undergoing percutaneous coronary revascularization.

Methods: We analyzed 2,767 patients with first NSTEMI from the Polish Registry of Acute Coronary Syndromes (PL-ACS) who underwent PCI. The patients were divided according to post-procedural culprit vessel TIMI (0-1: 90, 3.26\%; 2: 61, 2.20\%; 3: 2,616, 94.54\%).

Results: The following mortality values were obtained in TIMI 0-1, 2, and 3 groups, respectively: in-hospital, 12.22\%, 13.11\%, 1.72\% ( $p<0.0001)$; 1-month, 13.33\%, 13.11\%, 3.44\% ( $p<0.0001$ ); 12-month, 15.56\%, 16.39\%, 6.50\% ( $p<0.0001$ ); 36-month, 25.56\%, $21.31 \%$, $13.91 \%(p=0.0007)$. Mortality rates in patients with final TIMI $0-1$ and 2 were not significantly different. Optimal TIMI 3 was independently associated with baseline TIMI 2-3 (OR \pm $\pm 95 \%$ CI: 7.070 [4.35-11.82]), $p<0.0001$; higher ejection fraction (1.30 [1.03-1.63]), $p=0.0038$; and family history of coronary artery disease (2.83 [1.17-8.11]), $p=0.0294$. Type $C$ lesion, previous heart failure, and PCI without stenting independently predicted suboptimal TIMI 0-2.

Conclusions: Only achieving final TIMI 3 in IRA improves outcomes in NSTEMI patients treated with percutaneous coronary revascularization. The mortality rate of near-normal TIMI 2 is comparable to that of TIMI 0-1 after PCI. (Cardiol J 2016; 23, 4: 402-410)

Key words: acute coronary syndrome, infarct-related coronary artery, non-ST-segment elevation myocardial infarction, percutaneous coronary intervention

Address for correspondence: Jarosław Karwowski, MD, $2^{\text {nd }}$ Department of Coronary Artery Disease, Institute of Cardiology, ul. Spartańska 1, 02-637 Warszawa, Poland, tel/fax: +48 22 3434050/22 8449510, e-mail: karwowski.jarek@gmail.com 


\section{Introduction}

Registry data consistently show that non-ST-segment elevation myocardial infarction (NSTEMI) is the most common type of acute coronary syndrome [1]. Although in-hospital mortality is higher in patients with ST-segment elevation myocardial infarction (STEMI) than among those with NSTEMI, long-term follow-up showed worse outcomes in NSTEMI patients [1,2]. It is important to determine factors worsening prognosis in this constantly increasing group of patients. In the era of interventional cardiology, percutaneous coronary revascularization is the main method of treatment, which has been demonstrated to improve clinical outcomes [3, 4]. The goal of angioplasty is prompt restoration of normal flow in the infarct-related artery (IRA). Only post-procedural Thrombolysis in Myocardial Infarction (TIMI) flow grade 3 improves outcomes in patients with STEMI. Although it was shown that prognosis in patients with final TIMI flow grade 2 does not differ from that in patients with TIMI flow grade $0-1$, this conclusion was based on the results of outdated studies involving acute myocardial infarction (AMI) patients treated with thrombolytic therapy [5-7]. Thus, there is a paucity of data on the association between angiographic findings and clinical outcomes in patients with myocardial infarction (MI) without ST-segment elevation undergoing percutaneous coronary intervention (PCI). Moreover, clinical manifestations and predictors of suboptimal final coronary flow in this group, have not been investigated. Therefore, this study aimed to compare clinical characteristics, angiographic findings, and prognoses between PCI-treated NSTEMI patients with final TIMI $0-1$ and 2 and those with final TIMI 3 using data from the Polish Registry of Acute Coronary Syndromes (PL-ACS).

\section{Methods}

\section{Registry design}

The PL-ACS registry is an ongoing, nationwide, multicenter, prospective, observational study of hospitalized patients with the entire spectrum of acute coronary syndromes (ACS) in Poland designed to collect information regarding epidemiology, treatment, and outcomes. The method and results of the investigation of the first 100,193 patients have been described [8]. Hospitals in all Polish regions were invited to enter the registry if they had a cardiology unit, an internal medicine unit, or if they hospitalized $\geq 10$ patients with ACS per year. The detailed protocol with inclusion/ /exclusion criteria, methods and logistics, as well as definitions of all fields in the registry database was revised in May 2004 to comply with the Cardiology Audit and Registration Data Standards [9]. Only patients with confirmed ACS were enrolled in the registry. If, within the acute phase of MI, a patient was transferred to another hospital, both hospitals were required to complete the case report form, and these hospitalizations were subsequently linked together and analyzed as 1 case. Data were collected by skilled physicians who were in charge of each patient. After initial internal checks for missing or conflicting data, values outside the expected ranges were implemented in the software. Once a month, collected data were sent to the National Health Fund (NFZ), where they were crosschecked with standard hospital reports. The NFZ then transferred the data to the central database in the Silesian Center for Heart Diseases in Zabrze, Poland, where further checks were conducted. All-cause mortality data, including exact dates of death were obtained from government database (NFZ - the only and obligatory health insurer for all citizens of Poland). The PL-ACS database was linked to the death database with the use of the personal identification number unique for every patient in the registry. Data on in-hospital complications were collected by physicians who were taking care of the patients.

\section{Patients}

Between January and December 2008, a total of 4,125 patients with NSTEMI were treated with $\mathrm{PCI}$ and registered in the PL-ACS. The patients with prior MI, PCI, and coronary artery bypass grafting were excluded from the analysis. The remaining 2,767 patients were enrolled in this investigation.

\section{Definition and treatment}

To analyze the prognostic impact of final TIMI in IRA, patients were divided into three groups based on post-procedural TIMI: 0-1 (90 patients, $3.26 \%$ ), 2 (61 patients, $2.20 \%$ ), and 3 (2,616 patients, $94.54 \%$ ). TIMI grade flow is a scoring system from 0-3 referring to levels of coronary blood flow assessed during angiography: TIMI 0 flow (no perfusion) refers to the absence of any antegrade flow beyond a coronary occlusion; TIMI 1 flow (penetration without perfusion) is a faint antegrade coronary flow beyond the occlusion, with incomplete filling of the distal coronary bed; TIMI 2 flow (partial reperfusion) is a delayed or 
sluggish antegrade flow with complete filling of the distal territory; TIMI 3 is a normal flow which fills the distal coronary bed completely. NSTEMI was defined as (1) the absence of ST-segment elevation consistent with $\mathrm{MI} \geq 2 \mathrm{~mm}$ in adjacent chest leads and ST-segment elevation $\geq 1 \mathrm{~mm}$ in 2 standard leads and new left bundle branch block; and (2) positive cardiac necrosis markers. A coronary artery was considered an IRA (culprit) based on angiographic features (confirmed or suspected thrombus, ruptured or ulcerated plaque, TIMI flow grade $\leq 2$ ), electrocardiography recording, and echocardiographic findings. In-hospital and long-term mortalities were defined as death from all causes. Treatment strategy was considered invasive if coronary angioplasty was performed during the index hospitalization. Decisions related to treatment modalities (i.e., use of stents, intraaortic balloon pump, glycoprotein (GP) IIb/IIIa inhibitors, and angioplasty methods) were left to the discretion of attending physicians. This study was approved by appropriate institutional review boards.

\section{Statistical analysis}

Baseline characteristics were summarized as percentages for categorical variables and arithmetic means \pm standard deviations or medians and interquartile ranges for continuous variables. Continuous data distributions were analyzed with the Kolmogorov-Smirnov test. Baseline comparisons were made to test for differences between the groups using $\chi^{2}$ tests for frequency data and ANOVA (or the Kruskal-Wallis test) with Tukey's post-hoc method. To determine possible predictors of good TIMI flow after percutaneous transluminal coronary angioplasty (PTCA), variables were analyzed univariately. A multivariable logistic regression model was then developed using a directed stepwise approach with the following variables: age, sex, hypercholesterolemia, heart failure history, family history of coronary artery disease, left ventricular ejection fraction (LVEF), cardiogenic shock, GRACE score, pre-procedural TIMI (0-1 vs. 2-3), time to PTCA (from onset), stent utilization, and type $\mathrm{C}$ lesion. Factors that met the significance criteria of $\leq 0.05$ were retained in the final model. Curves of cumulative probability of death were constructed according to the Kaplan-Meier method, and cumulative event rates were compared by Wilcoxon test. Hazard ratio (HR) estimates with $95 \%$ confidence interval (CI) were calculated using Cox proportional hazard regression analysis which included all variables with p-values in the univariate analyses $<0.01$. The proportional hazards assumption was verified using graphical methods (log-log survivor function-by-time curve). All tests were 2 -tailed. A p-value $<0.05$ was considered statistically significant. Statistical testing was performed using SAS version 9.2 (SAS Institute, NC).

\section{Results}

\section{Baseline characteristics}

(medical history and risk factors)

Table 1 compares the risk factors and baseline characteristics among the 2,767 included patients. The patients with TIMI $0-1$ and 2 after PCI were older, but this difference was significant only between the TIMI 2 and TIMI 3 groups (68.3 vs. 64.3 years, $\mathrm{p}<0.05$ ). Moreover, patients with post-PCI TIMI $0-1$ had a significantly higher frequency of previous heart failure than those with TIMI 3.

\section{Clinical and electrocardiographic findings}

The patients with post-procedural TIMI 3 had a remarkably higher incidence of sinus rhythm compared to TIMI $0-1$ and 2 and a lower incidence of atrial fibrillation, the difference was significant only between TIMI 2 and TIMI 3 patients (Table 2 ). The Killip class $\geq 3$ on admission was more common in patients with TIMI $0-1$ after PCI than in TIMI 3 patients $(7.78 \%$ vs. $2.67 \%, \mathrm{p}=0.0094)$, as was the number of patients with a GRACE risk score $>140(47.78 \%$ vs. $35.42 \%, p=0.0162)$. The TIMI 2 group showed intermediate values, but with no significant differences.

\section{Angiographic and echocardiographic findings}

The non-optimal groups ( $0-1$ and 2$)$ had significantly higher frequencies of type $\mathrm{C}$ lesion and initial TIMI $0-1$ in IRA than patients with TIMI 3 after PCI (Table 3). GP IIb/IIIa receptor antagonists were used more frequently in patients with TIMI $\leq 2$. Patients with final TIMI 0-1 had significantly lower LVEF compared to both TIMI $2(44.2 \pm 9.6$ vs. $48.7 \pm 10.8$, $\mathrm{p}<0.05)$ and TIMI $3(44.2 \pm 9.6$ vs. $49.9 \pm 9.8$, $\mathrm{p}<0.05)$ groups.

The post-procedural TIMI flow grades according to baseline TIMI flow grades in IRA are presented in Figure 1.

\section{Clinical outcome}

Although in-hospital and long-term mortality rates did not differ between patients with final TIMI 
Table 1. Baseline characteristics and risk factors of study patients.

\begin{tabular}{|c|c|c|c|c|c|c|c|}
\hline \multirow[t]{2}{*}{ Parameters } & \multirow{2}{*}{$\begin{array}{l}\text { TIMI 0-1 } \\
(n=90)\end{array}$} & \multirow{2}{*}{$\begin{array}{l}\text { TIMI } 2 \\
(n=61)\end{array}$} & \multirow{2}{*}{$\begin{array}{c}\text { TIMI } 3 \\
(n=2616)\end{array}$} & \multirow[t]{2}{*}{$\mathbf{P}$} & \multicolumn{3}{|c|}{ P for pairwise TIMI } \\
\hline & & & & & $0-1$ vs. 2 & $0-1$ vs. 3 & 2 vs. 3 \\
\hline Age [years] & $66.8 \pm 10.9$ & $68.3 \pm 11.7$ & $64.3 \pm 11.4$ & 0.004 & 0.72 & 0.10 & 0.021 \\
\hline Female & $32.2(29)$ & $34.4(21)$ & $35.2(921)$ & 0.84 & 0.78 & 0.56 & 0.90 \\
\hline Body mass index $\left[\mathrm{kg} / \mathrm{m}^{2}\right]$ & $28.1 \pm 6.8$ & $28.4 \pm 4.2$ & $28.2 \pm 6.7$ & 0.98 & 0.98 & 0.99 & 0.99 \\
\hline Hypertension & $64.4(58)$ & $72.1(44)$ & $71.7(1876)$ & 0.32 & 0.32 & 0.13 & 0.94 \\
\hline Diabetes mellitus & $27.8(25)$ & $27.8(17)$ & $24.6(645)$ & 0.68 & 0.99 & 0.50 & 0.56 \\
\hline Hyperlipidemia & $31.1(28)$ & $32.8(20)$ & $41.5(1086)$ & 0.06 & 0.83 & 0.049 & 0.17 \\
\hline Never smoking & $47.8(43)$ & $47.5(29)$ & $40.9(1070)$ & 0.47 & 0.98 & 0.19 & 0.30 \\
\hline Previous angina & $5.6(5)$ & $11.5(7)$ & $8.0(210)$ & 0.42 & 0.23 & 0.39 & 0.34 \\
\hline Family history CAD & $3.3(3)$ & $6.6(4)$ & $9.2(241)$ & 0.13 & 0.44 & 0.056 & 0.48 \\
\hline Previous stroke & $2.2(2)$ & $1.6(1)$ & $2.4(63)$ & 0.92 & 1.00 & 1.00 & 1.00 \\
\hline Heart failure & $7.8(7)$ & $4.9(3)$ & $3.1(80)$ & 0.035 & 0.74 & 0.024 & 0.44 \\
\hline Peripheral vascular disease & $5.6(5)$ & $3.3(2)$ & $3.8(99)$ & 0.67 & 0.70 & 0.39 & 1.00 \\
\hline Renal failure & $4.4(4)$ & $11.5(7)$ & $5.6(146)$ & 0.13 & 0.12 & 0.824 & 0.08 \\
\hline Pulmonary disease & $2.2(2)$ & $3.3(2)$ & $3.0(79)$ & 0.90 & 1.00 & 0.66 & 0.71 \\
\hline
\end{tabular}

Data are presented as percentage (number) or mean \pm standard deviation; CAD — coronary artery disease; TIMI — Thrombolysis in Myocardial Infarction

Table 2. Clinical and electrocardiographic findings at admission.

\begin{tabular}{|c|c|c|c|c|c|c|c|}
\hline & \multirow[t]{2}{*}{ TIMI 0-1 } & \multirow[t]{2}{*}{ TIMI 2} & \multirow[t]{2}{*}{ TIMI 3} & \multirow[t]{2}{*}{$\mathbf{P}$} & \multicolumn{3}{|c|}{ P for pairwise TIMI } \\
\hline & & & & & $0-1$ vs. & $0-1$ vs. 3 & 2 vs. 3 \\
\hline \multicolumn{8}{|l|}{ Dominant type of symptoms } \\
\hline Typical chest pain & $92.2(83)$ & $91.8(56)$ & $93.0(2434)$ & 0.90 & 1.00 & 0.77 & 0.61 \\
\hline Dyspnea & $4.4(4)$ & $4.9(3)$ & $2.0(53)$ & 0.053 & 1.00 & 0.12 & 0.13 \\
\hline Symptoms-to-PCl > $24 \mathrm{~h}$ & $31.1(28)$ & $42.6(26)$ & $36.6(959)$ & 0.35 & 0.15 & 0.28 & 0.34 \\
\hline Symptoms-to-PCl > $72 \mathrm{~h}$ & $5.6(5)$ & $3.3(2)$ & $3.7(97)$ & 0.565 & 0.70 & 0.39 & 1.00 \\
\hline \multicolumn{8}{|l|}{ Ischemic changes on ECG } \\
\hline ST-segment depression & $46.7(42)$ & $34.4(21)$ & $45.2(1183)$ & 0.23 & 0.13 & 0.78 & 0.094 \\
\hline T-wave inversion & $18.9(17)$ & $27.9(17)$ & $23.8(623)$ & 0.42 & 0.19 & 0.28 & 0.46 \\
\hline \multicolumn{8}{|l|}{ Heart rhythm } \\
\hline Sinus rhythm & $87.8(79)$ & $88.5(54)$ & 95.1 (2488) & $<0.0001$ & 0.89 & 0.006 & 0.033 \\
\hline Atrial fibrillation & $7.8 \%(7)$ & $11.5(7)$ & $4.4(116)$ & 0.014 & 0.44 & 0.13 & 0.021 \\
\hline Heart rate [bpm] & $78.7 \pm 19.7$ & $76.4 \pm 17.3$ & $76.6 \pm 16.2$ & 0.49 & 0.66 & 0.46 & 0.99 \\
\hline Systolic BP [mm Hg] & $134.4 \pm 28.6$ & $137.5 \pm 23.2$ & $139.5 \pm 24.7$ & 0.13 & 0.73 & 0.13 & 0.81 \\
\hline Diastolic BP [mm Hg] & $79.2 \pm 16.3$ & $80.6 \pm 14.9$ & $81.8 \pm 13.6$ & 0.18 & 0.83 & 0.19 & 0.77 \\
\hline Killip class $\geq 3$ & $7.8(7)$ & $3.3(2)$ & $2.7(70)$ & 0.016 & 0.15 & 0.009 & 0.27 \\
\hline GRACE score $>140$ & $47.8(43)$ & $44.3(27)$ & $35.4(927)$ & 0.022 & 0.67 & 0.016 & 0.15 \\
\hline GRACE score [points] & $144.5 \pm 42.8$ & $138 \pm 36.8$ & $131.4 \pm 33.1$ & 0.0005 & 0.47 & 0.0008 & 0.29 \\
\hline CK-MB [mg/dL]* & $\begin{array}{c}55.5 \\
{[25.4-127.0]}\end{array}$ & $\begin{array}{c}44.0 \\
{[37.0-97.1]}\end{array}$ & $\begin{array}{c}39.0 \\
{[20-89.0]}\end{array}$ & 0.0359 & 0.81 & 0.0394 & 0.11 \\
\hline Serum creatinine $[\mathrm{mg} / \mathrm{dL}]^{*}$ & $\begin{array}{c}0.98 \\
{[0.84-1.20]}\end{array}$ & $\begin{array}{c}0.98 \\
{[0.86-1.26]}\end{array}$ & $\begin{array}{c}0.97 \\
{[0.81-1.17]}\end{array}$ & 0.30 & 0.45 & 0.63 & 0.14 \\
\hline Admission glucose $[\mathrm{mg} / \mathrm{dL}]^{*}$ & $\begin{array}{c}129.0 \\
{[96.0-164.9]}\end{array}$ & $\begin{array}{c}118.9 \\
{[105-160.0]}\end{array}$ & $\begin{array}{c}113.0 \\
{[98-143.0]}\end{array}$ & 0.08 & 0.97 & 0.10 & 0.10 \\
\hline
\end{tabular}

Data are presented as percentage (number) or mean \pm standard deviation; *Median, with 25th; 75th percentiles range in brackets;

$\mathrm{BP}$ - blood pressure, CK-MB — creatine kinase MB isoenzyme; ECG - electrocardiogram; PCI — percutaneous coronary intervention; TIMI - Thrombolysis in Myocardial Infarction 
Table 3. Angiographic, echocardiographic and laboratory findings.

\begin{tabular}{|c|c|c|c|c|c|c|c|}
\hline & \multirow{2}{*}{$\begin{array}{l}\text { TIMI 0-1 } \\
(n=90)\end{array}$} & \multirow{2}{*}{$\begin{array}{l}\text { TIMI } 2 \\
(n=61)\end{array}$} & \multirow{2}{*}{$\begin{array}{c}\text { TIMI } 3 \\
(\mathrm{n}=2616)\end{array}$} & \multirow[t]{2}{*}{$\mathbf{P}$} & \multicolumn{3}{|c|}{ P for pairwise TIMI } \\
\hline & & & & & $0-1$ vs. 2 & $0-1$ vs. 3 & 2 vs. 3 \\
\hline \multicolumn{8}{|l|}{ Culprit lesion } \\
\hline $\begin{array}{l}\text { Left anterior descending } \\
\text { artery }\end{array}$ & $26.7(24)$ & $29.5(18)$ & 34.7 (907) & 0.21 & 0.70 & 0.12 & 0.40 \\
\hline Circumflex coronary artery & $34.4(31)$ & $36.1(22)$ & $35.2(922)$ & 0.98 & 0.84 & 0.88 & 0.89 \\
\hline Right coronary artery & $35.6(32)$ & $32.8(20)$ & $28.2(737)$ & 0.24 & 0.86 & 0.13 & 0.43 \\
\hline Left main coronary artery & $2.2(2)$ & $1.6(1)$ & $1.8(47)$ & 0.78 & 1.00 & 0.68 & 0.93 \\
\hline Type C lesion & $41.1(37)$ & $36.1(22)$ & $20.8(544)$ & $<0.0001$ & 0.47 & $<0.0001$ & 0.015 \\
\hline \multicolumn{8}{|l|}{ Initial TIMI flow } \\
\hline TIMI 0-1 & $92.2(83)$ & $78.7(48)$ & $42.0(1098)$ & $<0.0001$ & 0.016 & $<0.0001$ & $<0.0001$ \\
\hline TIMI 2 & $2.2(2)$ & $14.7(9)$ & $27.9(732)$ & $<0.0001$ & 0.007 & $<0.0001$ & 0.022 \\
\hline TIMI 3 & $5.6(5)$ & $6.6(4)$ & $30.1(786)$ & $<0.0001$ & 1.00 & $<0.0001$ & $<0.0001$ \\
\hline $\begin{array}{l}\text { Multivessel coronary } \\
\text { disease }\end{array}$ & $56.7(51)$ & $49.2(30)$ & 47.7 (1249) & 0.24 & 0.37 & 0.09 & 0.82 \\
\hline $\begin{array}{l}\mathrm{PCl} \text { without coronary } \\
\text { stenting }\end{array}$ & $77.8(70)$ & $22.9(14)$ & $4.1(106)$ & $<0.0001$ & $<0.0001$ & $<0.0001$ & $<0.0001$ \\
\hline LVEF [\%] & $44.2 \pm 9.6$ & $48.7 \pm 10.8$ & $49.9 \pm 9.8$ & $<0.0001$ & 0.046 & 0.0001 & 0.72 \\
\hline Anti-glycoprotein Ilb/IIla & $12.2(11)$ & $19.7(12)$ & $5.7(148)$ & $<0.0001$ & 0.21 & 0.009 & $<0.0005$ \\
\hline Clopidogrel & $74 \%(82)$ & $90.2 \%(55)$ & $93.3 \%(2440)$ & $<0.0001$ & 0.17 & $<0.0001$ & 0.30 \\
\hline Ticlopidine & $4.4 \%(4)$ & $3.3 \%(2)$ & $1.8 \%(47)$ & 0.14 & 1.0 & 0.09 & 0.31 \\
\hline
\end{tabular}

Data are presented as percentage (number) or mean \pm standard deviation; LVEF — left ventricular ejection fraction; PCI — percutaneous coronary intervention; TIMI — Thrombolysis in Myocardial Infarction

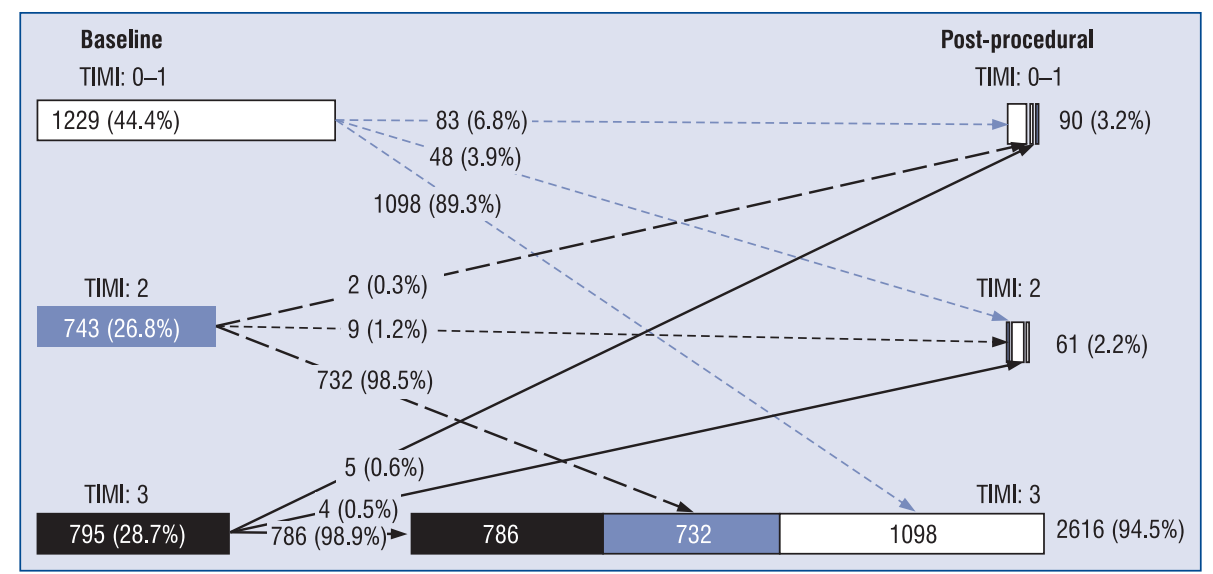

Figure 1. Post-procedural Thrombolysis in Myocardial Infarction (TIMI) flow grades according to baseline TIMI flow grades.

0-1 and 2 (Table 4), there were significant differences in in-hospital, 6-month, and 12-month mortality rates and borderline-significant differences in 24- and 36-month mortality rates between TIMI 2 and 3 patients. Survival curves after 3 years of observation showed significant difference between the TIMI 2 and 3 groups (Fig. 2).
As shown in Table 5, age, lower LVEF on admission, and higher GRACE risk score independently correlated with 6-month and 1-year mortalities. Further investigation revealed additional factors predicting all-cause mortality, such as renal failure after 2 years and renal failure, as well as lower body mass index after 3 years. 
Table 4. Short- and long-term mortality according to post-procedural Thrombolysis in Myocardial Infarction (TIMI) flow.

\begin{tabular}{|c|c|c|c|c|c|c|c|}
\hline \multirow[t]{2}{*}{ Mortality } & \multirow{2}{*}{$\begin{array}{c}\text { TIMI 0-1 } \\
\text { (n = 90) } \\
\%(n)\end{array}$} & \multirow{2}{*}{$\begin{array}{c}\text { TIMI } 2 \\
(n=61) \\
\%(n)\end{array}$} & \multirow{2}{*}{$\begin{array}{c}\text { TIMI } 3 \\
(n=2616) \\
\%(n)\end{array}$} & \multirow[t]{2}{*}{$\mathbf{P}$} & \multicolumn{3}{|c|}{ P for pairwise TIMI } \\
\hline & & & & & $0-1$ vs. 2 & $0-1$ vs. 3 & 2 vs. 3 \\
\hline In-hospital & $12.2(11)$ & $13.1(8)$ & $1.7(45)$ & $<0.0001$ & 0.87 & $<0.0001$ & $<0.0001$ \\
\hline 30-days & $13.3(12)$ & $13.1(8)$ & $3.4(90)$ & $<0.0001$ & 0.97 & $<0.0001$ & $<0.001$ \\
\hline 6-months & $15.6(14)$ & $16.4(10)$ & 6.5 (170) & $<0.0001$ & 0.89 & $<0.001$ & $<0.01$ \\
\hline 12-months & $15.6(14)$ & $18.0(11)$ & $8.5(222)$ & 0.0028 & 0.69 & $<0.02$ & $<0.01$ \\
\hline 24-months & $23.3(21)$ & $18.0(11)$ & $11.3(295)$ & 0.0007 & 0.43 & $<0.001$ & 0.10 \\
\hline 36-months & $25.6(23)$ & $21.3(13)$ & $13.9(364)$ & 0.0026 & 0.55 & $<0.002$ & 0.10 \\
\hline
\end{tabular}

Table 5. Independent predictors of 6-month, 12-month, 24-month and 36-month all-cause mortality.

\begin{tabular}{llcc}
\hline All-cause mortality & Predictors & Hazard ratio (95\% CI) & P \\
\hline 6-month & Age & $1.547(1.087-2.200)$ & 0.015 \\
& LVEF [\%] & $0.635(0.498-0.810)$ & 0.0003 \\
12-month & GRACE & $1.026(1.018-1.034)$ & $<0.0001$ \\
& Age & $1.544(1.152-2.069)$ & 0.004 \\
LVEF [\%] & GRACE & $0.685(0.554-0.846)$ & 0.0005 \\
& Renal failure in history & $1.024(1.017-1.031)$ & $<0.0001$ \\
& Age & $2.049(1.229-3.416)$ & 0.006 \\
$36-$ LVEF [\%] & $1.452(1.138-1.853)$ & 0.003 \\
& GRACE & $0.701(0.582-0.845)$ & 0.0002 \\
& Renal failure in history & $1.021(1.015-1.028)$ & $<0.0001$ \\
& Age & $2.336(1.466-3.721)$ & 0.0004 \\
& BMI & $1.463(1.181-1.811)$ & 0.0005 \\
& LVEF [\%] & $0.944(0.904-0.986)$ & 0.0101 \\
& GRACE & $0.634(0.534-0.752)$ & $<0001$ \\
& & $1.016(1.011-1.022)$ & $<0.0001$ \\
\hline
\end{tabular}

$\mathrm{Cl}$ — confidence interval; $\mathrm{BMI}$ — body mass index; LVEF — left ventricular ejection fraction

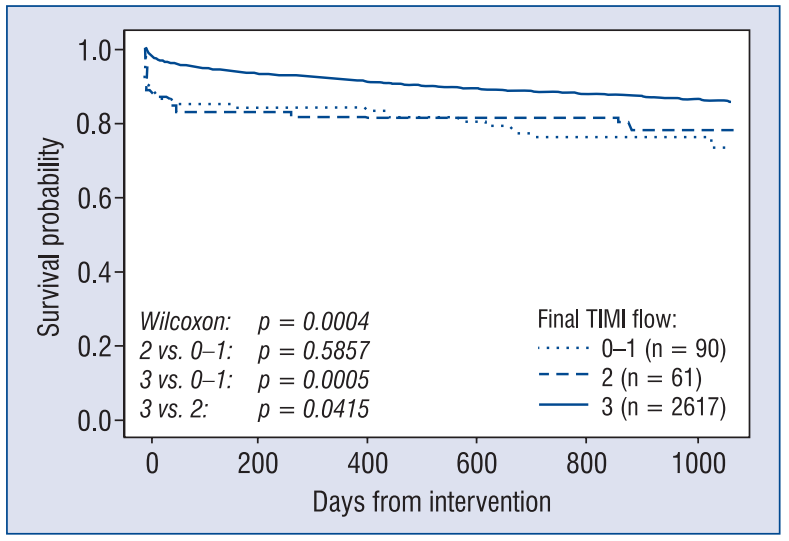

Figure 2. Kaplan-Meier 36-month survival curves according to postprocedural Thrombolysis in Myocardial Infarction (TIMI) flow.

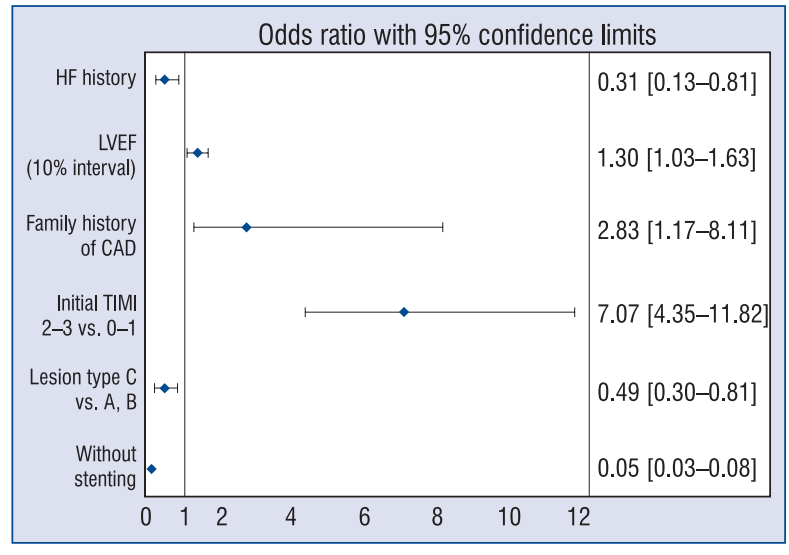

Figure 3. Independent predictors of postprocedural Thrombolysis in Myocardial Infarction (TIMI) flow in the infarct-related artery; HF — heart failure; LVEF — left ventricular ejection fraction; CAD coronary artery disease. 


\section{Clinical factors related to optimal TIMI 3 flow}

After adjustment for all clinical and angiographic characteristics, multivariate logistic regression demonstrated that optimal TIMI 3 was independently associated with baseline TIMI 2-3 (OR \pm 95 CI: 7.070 [4.35-11.82]), p < $<0.0001$; higher LVEF on admission (1.30 [1.03$-1.63]), \mathrm{p}=0.0038$; and family history of coronary artery disease (2.83 [1.17-8.11]), $\mathrm{p}=0.0294$ (Fig. 3). Type $\mathrm{C}$ lesion, heart failure in history, and $\mathrm{PCI}$ without stenting independently predicted suboptimal TIMI flow grade $0-2$.

\section{Discussion}

Our study demonstrated the relationship between post-PCI TIMI flow grade and outcomes in patients with NSTEMI. The main findings can be summarized as follows: 1) Only achieving the final TIMI 3 in the IRA improves the outcomes in NSTEMI patients treated with percutaneous coronary revascularization; 2) Interestingly, the mortality rate in patients with near-normal TIMI 2 was comparable to that in patients with TIMI 0-1 after PCI; 3) Multivariable analysis revealed that type $\mathrm{C}$ lesion, heart failure history, and PCI without stenting independently predicted the lack of optimal TIMI 3 after PCI.

To the best of our knowledge, this is the first study evaluating the clinical impact of final TIMI 2 on mortality in a large cohort of NSTEMI patients treated with PCI. The analysis shows no significant differences in hospital, 12-month, and 36 -month outcomes between patients with final TIMI $0-1$ and 2. Furthermore, despite the lack of significant differences in the proportion of the GRACE risk score and incidence of symptoms of heart failure on admission between patients with post-procedural TIMI 2 and 3, the TIMI 2 group had significantly higher in-hospital, 6-month, and 12 -month mortalities. In an earlier study, patients with TIMI 2 had mortality rates intermediate to those of patients with TIMI $0-1$ and TIMI 3 [10]. However, it is difficult to compare these results as the cited study included a heterogeneous group of STEMI, NSTEMI, and unstable angina patients, with NSTEMI patients constituting $<1 / 3$ of the study population.

Interestingly, although differences in in-hospital and 1-year mortalities between patients with final TIMI 2 and 3 in IRA were significant, the 24-month and 36-month mortalities were only at the edge of statistical significance. An attempt to investigate this discrepancy with multivariable analysis of mortality revealed additional non-cardiac predictors of death identified during 24- and 36-month follow-up, such as renal failure and body mass index. This might be the main reason why statistically significant differences in mortality after 1 year were not observed. Because this study evaluated all-cause mortality, non-cardiac factors may have somewhat reduced the significance of the differences in all-cause mortality more than 1 year after NSTEMI.

Predictors of non-optimal coronary flow after percutaneous coronary revascularization in NSTEMI patients have not been described so far. In the present report, lower LVEF at presentation and previous left ventricular dysfunction were associated with a higher likelihood of poor final TIMI in IRA. Heart failure is associated with higher left ventricular end-diastolic pressure and reduced coronary perfusion pressure. These might cause more severe damage to microvascular circulation and interstitial edema, which in effect increases the risk of no-reflow syndrome. These findings are in agreement with the results of a previous study involving STEMI patients, which demonstrated that low LVEF and advanced Killip class on admission were associated with higher prevalence of suboptimal TIMI 0-2 in STEMI patients undergoing primary angioplasty [10].

Type $\mathrm{C}$ lesion, determined according to the classification of American College of Cardiology/ /American Heart Association, independently predicted suboptimal final TIMI $0-1$ and 2 . It has been shown that type A lesion angioplasty is associated with $90 \%$ probability of success versus only $60 \%$ for type C [11]. In our report, the incidence of type $\mathrm{C}$ lesion was approximately 2 -fold higher in TIMI $0-1$ and 2 groups than in the TIMI 3 group. Furthermore, pre-procedural TIMI 0-1 was associated with a higher likelihood of suboptimal coronary flow after angioplasty. Initial total artery occlusion may reflect higher clot burden, the lack of spontaneous clot lysis, and higher amounts of thrombi and plaque material, leading to distal embolization. These findings are in agreement with previous studies on patients with STEMI undergoing PCI [12-14]. More advanced coronary lesions in combination with poorer initial flow in the IRA in TIMI $0-1$ and 2 groups were responsible for the higher rate of revascularization failure.

Patients with post-procedural TIMI $0-1$ and 2 were older than those in the TIMI 3 group. Aging is associated with significant cardiovascular structural changes, including endothelial and vascular smooth muscle dysfunction, diffuse 
coronary atherosclerosis, increased coagulation factors, severe vascular calcification, and disrupted microcirculation $[15,16]$, which makes the heart less resistant to ischemia and more prone to distal embolization during coronary angioplasty. According to the PAMI trial, age above 70 years is associated with suboptimal final coronary flow in STEMI patients [12].

In patients undergoing acute coronary intervention following MI, distal embolization caused by thrombi and plaque material is an important additional mechanism, which may induce revascularization failure. Downstream atheroembolization causes further blockage of structurally and functionally changed cardiac arterioles and capillaries. It was reported that GP IIb/IIIa inhibitors reduce the clot burden at the epicardial coronary level and improve microvascular flow $[17,18]$. In our study, GP IIb/IIIa receptor antagonists were used more frequently in TIMI $0-1$ and 2 groups. It is known that intravenous administration of these antagonists not only blocks the final common pathway for platelet aggregation and prevents aggregation regardless of the initial activating stimulus, but could also enable lysis of an acutely formed platelet thrombus [19, 20]. Although proximal and distal mechanical protection devices and thrombectomy catheters may also prevent from distal embolization, their effects on myocardial perfusion and clinical outcome in patients with AMI remain unclear and controversial [21].

Apart from GP IIb/llla inhibitors administration, early dual antiplatelet and appropriate anticoagulant therapies may reduce clot burden and improve coronary flow. Previous studies based on STEMI population showed benefits from intracoronary administration of adenosine or verapamil in prevention of revascularization failure by reducing microvascular reperfusion damage, but there is lack of data in NSTEMl $[22,23]$. It has been reported that patients with metabolic syndromes [24] and hyperglycemia [25] are more likely to develop endothelial dysfunction and revascularization failure, therefore attention to blood sugar in patients with MI is important. Direct stenting compared with conventional stenting results in significantly greater rates of final TIMI3 flow in STEMI [26]. Further clinical research in pharmacological and procedural techniques improving acute angiographic outcome in NSTEMl patients is needed together with industry involvement.

The precise mechanism by which final TIMI 2 flow in NSTEMI patients undergoing primary PCI has impact on higher mortality is difficult to explain based on our research. Probably, not full restoration of coronary flow do not guarantee optimal myocardial perfusion and do not save as much as possible viable myocardium. On the other hand, higher incidence of final TIMI 2 flow among high-risk patients (older age, lower baseline TIMI flow, lower LVEF) suggests that TIMI 2 flow may be a surrogate marker of these high-risk groups. Taking all those observations into account, TIMI 2 patients require more exact control and more aggressive treatment after angioplasty.

\section{Limitations of the study}

The first limitation of this study is its retrospective nature. Furthermore, the culprit artery was determined by cardiologists in the catheterization laboratories (using electrocardiographic, angiographic and echocardiographic findings), and the selection of the IRAs among the patients with multivessel disease might have differed between operators. Patients with previous MIs, percutaneous interventions, and coronary artery bypass grafting were excluded from the study because the culprit vessel in these subjects might have been incorrectly interpreted due to chronic total occlusion. Finally, there is a lack of data regarding detailed angiographic features, such as myocardial blush grade and thrombus burden.

\section{Conclusions}

This study demonstrates that achieving "near-normal" post-procedural TIMI flow grade 2 does not improve prognosis in NSTEMI patients who underwent PCI. Only final TIMI flow grade 3 should be considered an indication of successful revascularization. Patients with a history of heart failure, type $\mathrm{C}$ lesion in infarct-related coronary artery, and those treated without stenting are at a greater risk of suboptimal TIMI 0-2. Every effort should be made to achieve complete artery patency.

\section{Acknowledgments}

We thank all the physicians and nurses who participated in PL-ACS, members of the Expert Committee, and staff members of the National Health Fund of Poland for their logistic support. The PL-ACS Expert Committee includes: Lech Poloński (Chairman), Mariusz Gąsior (Co-chairman), Marek Gierlotka (Co-chairman), and Zbigniew Kalarus (Co-chairman), Zabrze, Poland; Andrzej Cieśliński, Poznań, Poland; Jacek Dubiel, Krakow, Poland; Robert Gil, Grzegorz Opolski, 
Witold Rużyło, Warsaw, Poland; Michał Tendera, Katowice, Poland; and Marian Zembala, Zabrze, Poland.

\section{Conflict of interest: None declared}

\section{References}

1. Fox K, Eagle K, Gore J, Steg P, Anderson FA. The Global Registry of Acute Coronary Events, 1999 to 2009 - GRACE. Heart, 2010; 96: 1095-1101.

2. Polonski L, Gasior M, Gierlotka M et al. A comparison of ST elevation versus non-ST elevation myocardial infarction outcomes in a large registry database: Are non-ST myocardial infarctions associated with worse long-term prognoses? Int J Cardiol, 2011; 152: 70-77.

3. Weaver $\mathrm{W}$, White H, Wilcox R et al. Comparisons of characteristics and outcomes among women and men with acute myocardial infarction treated with thrombolytic therapy. GUSTO-I investigators. JAMA, 1996; 275: 777-782.

4. Zijlstra F, Hoorntje J, de Boer M et al. Long-term benefit of primary angioplasty as compared with thrombolytic therapy for acute myocardial infarction. N Engl J Med, 1999; 341: 1413-1419.

5. Karagounis L, Sorensen S, Menlove R et al. Does thrombolysis in myocardial infarction (TIMI) perfusion grade 2 represent a mostly patent artery or a mostly occluded artery? Enzymatic and electrocardiographic evidence from the TEAM-2 study. Second Multicenter Thrombolysis Trial of Eminase in Acute Myocardial Infarction. J Am Coll Cardiol, 1992; 19: 1-10.

6. Anderson J, Karagounis L, Becker L, Sorensen S, Menlove R. TIMI perfusion grade 3 but not grade 2 results in improved outcome after thrombolysis for myocardial infarction. Ventriculographic, enzymatic, and electrocardiographic evidence from the TEAM-3 study. Circulation, 1993; 87: 1829-1839.

7. Anderson J, Karagounis L, Califf R. Metaanalysis of five reported studies on the relation of early coronary patency grades with mortality and outcomes after acute myocardial infarction. Am J Cardiol, 1996; 78: 1-8.

8. Polonski L, Gasior M, Gierlotka M et al. Polish Registry of Acute Coronary Syndromes (PL-ACS). Characteristics, treatments and outcomes of patients with acute coronary syndromes in Poland. Kardiol Pol, 2007; 65: 861-872.

9. Flynn M, Barrett C, Cosio F et al. The Cardiology Audit and Registration Data Standards (CARDS), European data standards for clinical cardiology practice. Eur Heart J, 2005; 26: 308-313.

10. Ndrepepa G, Mehilli J, Schulz S et al. Prognostic significance of epicardial blood flow before and after percutaneous coronary intervention in patients with acute coronary syndromes. J Am Coll Cardiol, 2008; 52: 512-517.

11. Pompa J, Gibson C. Qualitative and Quantitative Angioplasty. In: Topol EJ ed. Textbook of Interventional Cardiology. Saunders, Elsevier Science 2003: 827-846.

12. Mehta R, Harjai K, Cox D et al. Clinical and angiographic correlates and outcomes of suboptimal coronary flow in patients with acute myocardial infarction undergoing primary percu- taneous coronary intervention. J Am Coll Cardiol, 2003; 42: 1739-1746.

13. Cura F, L'Allier P, Kapadia S et al. Predictors and prognosis of suboptimal coronary blood flow after primary coronary angioplasty in patients with acute myocardial infarction. Am J Cardiol, 2001; 88: 124-128.

14. Parodi G, Valenti R, Carrabba N et al. Long-term prognostic implications of nonoptimal primary angioplasty for acute myocardial infarction. Catheter Cardiovasc Interv, 2006; 68: 50-55.

15. Hogikyan R, Supiano M. Arterial alpha-adrenergic responsiveness is decreased and SNS activity is increased in older humans. Am J Physiol, 1994; 266: E717-E724.

16. Lowe G, Rumley A, Woodward M, et al. Epidemiology of coagulation factors, inhibitors and activation markers: The Third Glasgow MONICA Survey I. Illustrative reference ranges by age, sex, and hormone use. Br J Haematol, 1997; 97: 775-784.

17. De Lemos J, Antman E, Gibson C et al. Abciximab improves both epicardial flow and myocardial reperfusion in ST elevation myocardial infarction. Observations from the TIMI 14 trial. Circulation, 2000; 101: 239-243.

18. Neumann F, Blasini R, Schmitt C et al. Effect of glycoprotein IIb/IIIa receptor blockade on recovery of coronary flow and left ventricular function after the placement of coronary-artery stents in acute myocardial infarction. Circulation, 1998; 98: 2695-2701.

19. Collet J, Montalescot G, Lesty C et al. Effects of Abciximab on the architecture of platelet-rich clots in patients with acute myocardial infarction undergoing primary coronary intervention. Circulation, 2001; 103: 2328-2331.

20. Collet J, Montalescot G, Lesty C, Weisel J. A structural and dynamic investigation of the facilitating effect of glycoprotein $\mathrm{IIb} / \mathrm{III}$ inhibitors in dissolving platelet-rich clots. Circ Res, 2002; 90: 428-434

21. De Luca G, Suryapranata H, Stone G, Antoniucci D et al. Adjunctive mechanical devices to prevent distal embolization in patients undergoing mechanical revascularization for acute myocardial infarction: A meta-analysis of randomized trials. Am Heart J, 2007; 153: 343-353.

22. Taniyama $\mathrm{Y}$, Ito $\mathrm{H}$, Iwakura $\mathrm{K}$ et al. Beneficial effect of intracoronary verapamil on microvascular and myocardial salvage in patients with acute myocardial infarction. J Am Coll Cardiol, 1997; 30: 1193-1199.

23. Marzilli M, Orsini E, Marraccini P et al. Beneficial effects of intracoronary adenosine as an adjunct to primary angioplasty in acute myocardial infarction. Circulation, 2000; 101: 2154-2159.

24. Tartan Z, Ozer N, Uyarel H et al. Metabolic syndrome is a predictor for an ECG sign of no-reflow after primary PCI in patients with acute ST-elevation myocardial infarction. Nutr Metab Cardiovasc Dis, 2008; 18: 441-447.

25. Iwakura K, Ito H, Ikushima M et al. Association between hyperglycemia and the no-reflow phenomenon in patients with acute myocardial infarction. J Am Coll Cardiol, 2003; 41: 1-7.

26. Dziewierz A, Siudak Z, Rakowski T et al. Impact of direct stenting on outcome of patients with ST-elevation myocardial infarction transferred for primary percutaneous coronary intervention (from the EUROTRANSFER registry). Catheter Cardiovasc Interv, 2014; 84: 925-931. 